\title{
Systematic Review and Meta-Analysis of Randomized Controlled Trials on the Effect of SGLT2 Inhibitor on Blood Leptin and Adiponectin Level in Patients with Type 2 Diabetes
}

\author{
Authors \\ Peili Wu*, Weiheng Wen *, Jitong Li *, Jie Xu, Min Zhao, Hong Chen, Jia Sun
}

\begin{abstract}
Affiliation
Department of Endocrinology, Zhujiang Hospital, Southern Medical University, Guangzhou, P. R. China
\end{abstract}

Key words

hypoglycemic drugs, adipokines, type 2 diabetes mellitus

$\begin{array}{lr}\text { received } & 25.09 .2018 \\ \text { accepted } & 12.06 .2019\end{array}$

Bibliography

DOI https://doi.org/10.1055/a-0958-2441

Horm Metab Res 2019; 51: 487-494

(c) Georg Thieme Verlag KG Stuttgart · New York

ISSN 0018-5043

Correspondence

Dr. Hong Chen

Department of Endocrinology

Zhujiang Hospital

Southern Medical University

Guangzhou

P. R. China

chenhong123@smu.edu.cn

Dr. Jia Sun

Department of Endocrinology

Zhujiang Hospital

Southern Medical University

510280 Guangzhou

P. R. China

Tel./Fax:+862061643195

sunjia@smu.edu.cn $\circledast$ Supplementary Material for this article is available online at http://www.thieme-connect.de/products

\section{ABSTRACT}

Sodium glucose cotransporter 2 (SGLT2) inhibitors are a new kind of hypoglycemic drugs that improve glucose homeostasis by inhibiting renal glucose reabsorption. Recent studies have shown that SGLT2 inhibitors can also mediate body metabolism through regulation of adipokines level, but the effects of SGLT2 inhibitors on the concentration of adipokines (leptin and adiponectin) remains controversial. This meta-analysis was set out to evaluate the changes in circulating leptin and adiponectin levels in patients with type 2 diabetes mellitus (T2DM) receiving SGLT2 inhibitors therapy. Ten randomized controlled trials (RCTs), that evaluated the effects of SGLT2 inhibitors on blood leptin and adiponectin levels in patients with type 2 diabetes, were identified by performing a systematic search of Pubmed, Embase, Cochrane, and Web of science databases through July 2018. Data were calculated using a random-effects model and presented as standardized mean difference (SMD) and $95 \%$ confidence interval $(\mathrm{Cl})$. Compared with placebo, treatment with SGLT2 inhibitors contributed to a decreased circulating leptin levels (SMD $-0.29,95 \% \mathrm{Cl}-0.56,-0.03)$ and an increased circulating adiponectin levels (SMD 0.30, 95\% CI 0.22, 0.38). SGLT2 inhibitor treatment was associated with decreased circulating leptin levels and increased circulating adiponectin levels, which might contribute to the beneficial effects of SGLT2 inhibitors on metabolic homeostasis.

\section{Introduction}

T2DM is one of the most prevalent metabolic disorders around the world [1]. The characters of T2DM, insulin resistance, relative insulin deficiency, and gradual pancreatic $\beta$ cell dysfunction are partly attributed to dysregulation of adipokines derived from adipose tis-

\footnotetext{
${ }^{*}$ Contributed equally to this work.
}

sue [2]. Since the discovery of leptin, the first identified adipokine in the 1990s, the list of adipokines has been increasing constantly and now includes some biological molecules such as adiponectin, resistin, visfatin, and IL- 6 that have essential roles in glucose and lipid metabolism [3-5]. Leptin, a 16 kDA classical adipokine, is known to suppress food intake and mediate energy homeostasis including glucose and lipid metabolism [4]. Several gene polymorphisms of leptin have been reported to be associated with T2DM 
$[6,7]$. In addition, leptin treatment could not only improve insulin resistance in patients, but also suppress liver gluconeogenesis as well as fasting hyperglycemia in diabetic mice [8]. The serum level of leptin is elevated paradoxically in obesity, which is due to the increased fat mass as leptin is synthesized and secreted by adipocytes [9], and this high level of leptin itself may induce leptin resistance and thus result in decreasing the ability of glucose utilization and interfering glucose metabolism. The another adipocytokine, adiponectin, is also an adipose-derived secretory protein, and plays a pivotal role in insulin signal and energy metabolism as well [10]. Adiponectin is well known to reduce glucose production in liver and suppress TNF- $\alpha$ signal that contributed to insulin resistance [11]. Meanwhile, it is established that adiponectin is negatively correlated with body weight, percentage of body fat and basal plasma insulin [12]. All the above evidence demonstrates that leptin and adiponectin may be an important factor for T2DM development. Also, these two kinds of adipokines have been reported to have cardiovascular protection [13].

SGLT2 inhibitors, an emerging kind of antidiabetic drugs, decrease plasma glucose by promoting urinary glucose excretion [14]. SGLT2 inhibitors are also reported to improve glycemic control as well as reduce risk of cardiovascular diseases $[15,16]$. To explore how SGLT2 inhibitors improve the metabolic disturbances in type 2 diabetes patients, certain researches have been performed to study the changes in adipokines after SGLT2 inhibitors administration in patients with type 2 diabetes. However, previous results have been inconsistent [17-20], and the association between adipokines and SGLT2 inhibitors remains unclear. We therefore performed this meta-analysis with currently available clinical data to further evaluate the association between the treatment of SGLT2 inhibitor and circulating leptin or adiponectin concentrations in patients with T2DM.

\section{Materials and Methods}

\section{Search strategy}

Databases such as Pubmed, Embase, Cochrane, and Web of science were systematically searched without limitations in languages between January 1980 and April 2019. Specific terms like "Diabetes Mellitus, Type 2" or "type 2 diabetes mellitus" or "type 2 diabetes" or "Diabetes" or "diabetic mellitus" and "sglt2" or "sglt-2" or "sodium-glucose cotransporter 2 inhibitors" or "dapagliflozin" or "canagliflozin" or "empagliflozin" or "ipragliflozin" or "tofogliflozin" or "luseogliflozin" and "adipocytokines" or "adipokines" or "leptin" or "obese protein" or "ob protein" or "obese gene product" or "adiponectin" or "Adipocyte Complement Related Protein 30 kDa" or "apM 1 Protein" or "ACRP30 Protein" were selected to be the key words to sift out studies, which might be potentially relevant. Each reference lists of related meta-analysis or systemic review were also searched manually for identifying other eligible studies.

\section{Inclusive and exclusive criteria}

All the subjects included in this study had to be qualified with the following criteria: (1) RCTs of SGLT2 inhibitor treatment compared to placebo in type 2 diabetes patients as a monotherapy or add-on treatment and (2) The change of serum leptin and adiponectin level from baseline of each subject in comparative groups should be available. Studies were excluded if they were nonrandomized trials, if they included patients with type 1 diabetes, or if they provide inadequate data about quantitative outcomes.

\section{Data extraction}

Two investigators (PLW and JTL) independently scrutinized each article without knowing the findings of each other, and reached a consensus including time of study, author names, sample sizes, circulating leptin and adiponectin concentrations, and background therapy. Overlapping data sets or study groups were excluded.

\section{Quality assessment}

Quality assessment of the identified randomized controlled trials was using Cochrane risk of bias tool based on the following items: sequence generation, allocation concealment, blinding of participants and personnel, blinding of outcome assessment, incomplete outcome data, selective reporting and other bias. Two investigators (PLW and JTL) independently analyzed and judged each specific domain as low risk, unclear risk and high risk.

\section{Data analysis}

The statistical analysis was conducted using STATA 12.0. To assess the association between SGLT2 inhibitors use and the change of serum leptin and adiponectin level from baseline, standard mean difference and $95 \%$ confidence intervals for change of serum leptin and adiponectin concentrations in type 2 diabetes subjects were calculated, and combined by performing fix or random-effect model. Higgins $1^{2}$ test were conducted to estimate heterogeneity among studies. $A \mathrm{I}^{2}$-value $>50 \%$ was taken as a high level of heterogeneity, and the random effects model was performed to pool the size effect. If $\mathrm{I}^{2}$-value $\leq 50$ was defined as a low level of heterogeneity, and the fix effects model were used for the analysis. To evaluate the sources of heterogeneity, we implemented subgroup analysis stratified by age, BMI and treatment duration. Sensitive analysis was performed to assess the influence of each single study on the change of circulating leptin and adiponectin levels. Publication bias were evaluated by Begg's test, where a p-value less than 0.1 was taken as evidence of small study effects.

\section{Compliance with ethics guidelines}

This article is based on previously conducted studies and does not contain any studies with human participants or animals performed by any of the authors.

\section{Results}

\section{Meta-analysis of the association of adipokines and T2DM}

The search strategy first selected 132 articles. According to the inclusive and exclusive criteria, 1988 subjects of 10 random placebo-controlled studies were acquired ( $\bullet$ Fig. 1) $[17,20-28]$, and the characteristics of these studies are given in $\triangleright$ Table 1 . The mean age of study cases was 57.8 years, the mean $\mathrm{HbA} 1 \mathrm{c}$ was $64.92 \mathrm{mmol} /$ $\mathrm{mol}$, and the mean intervention duration was 20.2 weeks. Trial quality was determined using Cochrane risk of bias tool ( $\bullet$ Fig. 2 ). 


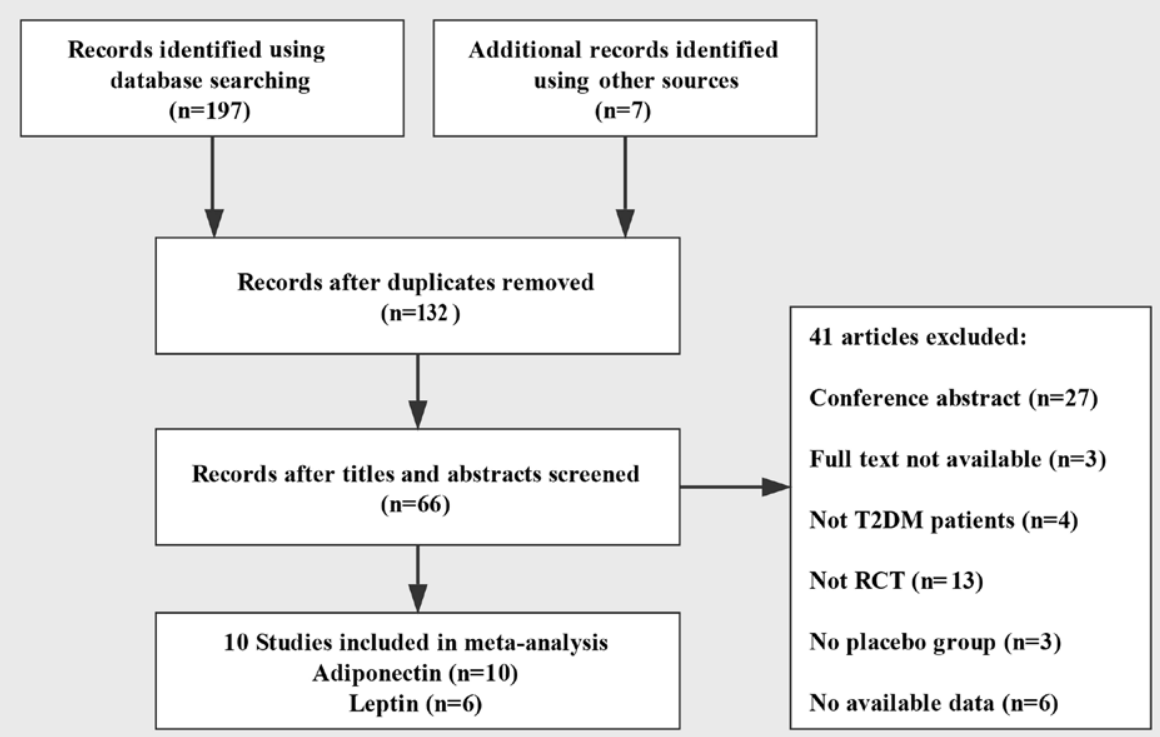

- Fig. 1 Flowchart of the selection of eligible studies.

\section{Association between SGLT2 inhibitor treatment and blood leptin levels}

Six studies compared circulating leptin levels between the SGLT2 inhibitors and placebo groups, and a total numbers of 1045 cases were identified for this evaluation. Compared with placebo group, use of SGLT2 inhibitors decreased leptin levels (SMD - 0.29, $95 \%$ $\mathrm{Cl}-0.56,-0.03 ; \mathrm{p}=0.032$ ) ( Fig. 3 ). The $\mathrm{I}^{2}$-value was $77.7 \%$, which implied a high level of heterogeneity among studies.

\section{Subgroup analysis}

Subgroup analysis stratified by age, BMI, and treatment duration was implemented, and the results are displayed in $>$ Table 2 . As for leptin levels, in subgroup of age $<60$ years, $\mathrm{BMI}<30 \mathrm{~kg} / \mathrm{m}^{2}$, and treatment duration $\geq 24$ weeks, patients receiving SGLT2 inhibitors therapy showed lower serum leptin levels than that of placebo group. No significant difference was observed in subgroup of age $\geq 60$ years, $B M I \geq 30 \mathrm{~kg} / \mathrm{m}^{2}$, and treatment duration $<24$ weeks in this analysis.

\section{Sensitivity analysis and publication bias}

To determine the influence of each single study on the overall results, we evaluated the stability of results with the use of a kick-out strategy. This method determines whether pooled values had significant fluctuation with the exclusion of each individual study. Sensitivity analysis displayed that after excluding of any study did not change the pooled result of this analysis (data not shown). In addition, after removal of the study conducted by Avogaro, the $\mathrm{I}^{2}$-value was decreased to 0 . The sample size of Avogaro study was less than 50 , and this study might be the source of heterogeneity among studies [17]. Begg's test suggested no certain evidence of publication bias, with a p-value of 0.536 ( $\vee$ Fig. 1S).

\section{Association between SGLT2 inhibitor treatment and blood adiponectin levels}

Ten randomized controlled studies including 1988 patients were suitable for analysis of the association between SGLT2 inhibitor therapy and circulating adiponectin levels. The result revealed that treatment of SGLT2 inhibitor was significantly elevated the blood adiponectin levels compared to placebo (SMD 0.30, $95 \% \mathrm{Cl} 0.22$, 0.38 ; $p<0.001$ ) ( Fig. 4). The $\mathrm{I}^{2}$-value was $66.4 \%$, suggesting a high level of heterogeneity among studies.

\section{Subgroup analysis}

As for adiponectin levels, a statistically significant increase was also found in subgroup of age $<60$ years and $\mathrm{BMI}<30 \mathrm{~kg} / \mathrm{m}^{2}$, but the study remained with high heterogeneity ( $\triangleright$ Table 2 ). No significant difference was observed in subgroup of age $\geq 60$ years and $\mathrm{BMI} \geq 30 \mathrm{~kg} / \mathrm{m}^{2}$ in this analysis. Additionally, despite the higher heterogeneity, patients in subgroup of treatment duration $\geq 24$ weeks or not showed increased serum adiponectin levels.

\section{Sensitivity analysis and publication bias}

In the meta-analysis of the association of SGLT2 inhibitor therapy and leptin concentration, the result did not change significantly after removal of any study from the pooled research, which indicated a stable model of our analysis. Also, after removal of the study conducted by Avogaro, the $\mathrm{I}^{2}$-value was decreased from $66.4 \%$ to $58.3 \%$. Begg's test indicated no statistically significant publication bias, with a p-value of 0.216 ( $\nabla$ Fig. 2 S).

\section{Discussion}

Recent studies have shown that use of SGLT2 inhibitor can improve the insulin resistance $[29,30]$, which could be explained by the decrease of glucose toxicity in response to induced urinary glucose 


\begin{tabular}{|c|c|c|c|c|c|c|c|c|}
\hline 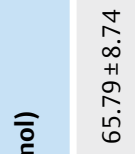 & 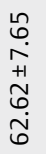 & 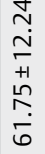 & $\begin{array}{l}8 \\
0 \\
\infty \\
+1 \\
\infty \\
0 \\
0 \\
0\end{array}$ & 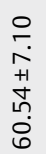 & $\begin{array}{l}\text { r } \\
\text { in } \\
+1 \\
\stackrel{1}{0} \\
\stackrel{0}{0} \\
\stackrel{1}{n}\end{array}$ & $\begin{array}{l}\text { Pे } \\
\dot{a} \\
+1 \\
\\
\hat{0}\end{array}$ & \begin{tabular}{l} 
กิ \\
$\infty$ \\
+1 \\
$\stackrel{+}{N}$ \\
\multirow{0}{0}{} \\
$\infty$
\end{tabular} & 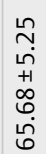 \\
\hline 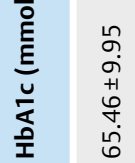 & $\begin{array}{l}8 \\
0 \\
\infty \\
+1 \\
+ \\
6 \\
m \\
0\end{array}$ & 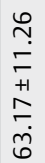 & $\begin{array}{l}0 \\
\infty \\
\\
+1 \\
\hat{\sigma} \\
\dot{0} \\
0\end{array}$ & $\begin{array}{l}\text { ro } \\
\stackrel{+}{+} \\
+1 \\
\stackrel{1}{\infty} \\
\dot{0}\end{array}$ & $\begin{array}{l}\text { ᄋ } \\
\text { ஸn } \\
+1 \\
\infty \\
\infty \\
\infty \\
\infty\end{array}$ & $\begin{array}{l}\stackrel{N}{~} \\
\infty \\
+1 \\
\infty \\
\stackrel{+}{N} \\
\end{array}$ & $\begin{array}{l}\tilde{F} \\
\infty \\
+1 \\
+1 \\
0 \\
0 \\
0 \\
0\end{array}$ & $\begin{array}{l}0 \\
+ \\
\text { in } \\
+1 \\
\stackrel{1}{m} \\
\dot{0}\end{array}$ \\
\hline 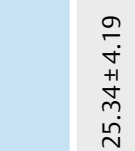 & $\begin{array}{l}\text { fे } \\
\text { in } \\
+1 \\
\text { ñ } \\
\text { ò } \\
\text { m. }\end{array}$ & $\begin{array}{l}\bar{\sigma} \\
\dot{+} \\
+1 \\
+ \\
\dot{+} \\
\dot{m}\end{array}$ & $\begin{array}{l}\text { oे } \\
\dot{m} \\
+1 \\
\stackrel{+}{+} \\
\stackrel{\dot{n}}{N}\end{array}$ & $\begin{array}{l}\text { బ } \\
\text { m } \\
+1 \\
\infty \\
\infty \\
\text { ஸे }\end{array}$ & $\begin{array}{l}\text { m. } \\
m \\
m \\
+1 \\
\mathscr{2} \\
\dot{\sim} \\
\dot{\sim}\end{array}$ & $\begin{array}{l} \\
\infty \\
\dot{m} \\
+1 \\
\stackrel{+}{+} \\
\dot{\varphi}\end{array}$ & $\begin{array}{l}= \\
\dot{+} \\
+1 \\
8 \\
\dot{0} \\
\dot{v}\end{array}$ & $\begin{array}{l}\check{m} \\
\dot{m} \\
\stackrel{+}{n} \\
\stackrel{n}{\sim}\end{array}$ \\
\hline
\end{tabular}

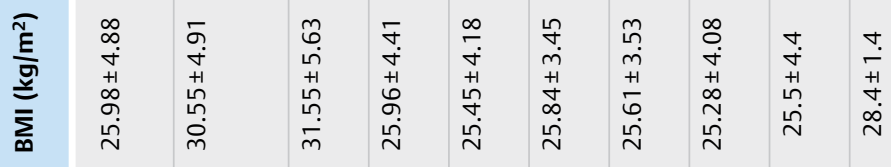

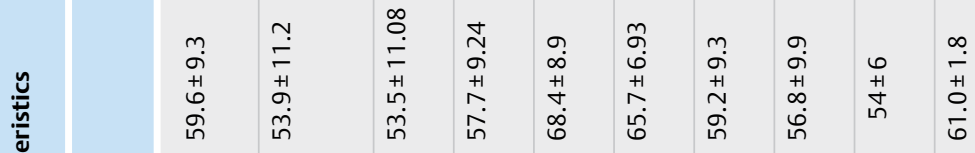

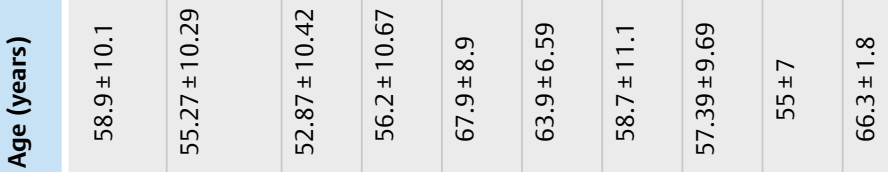

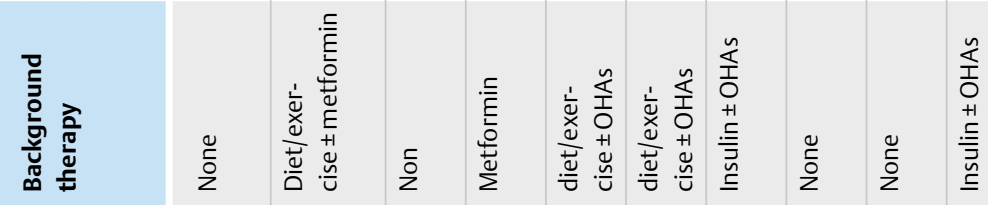

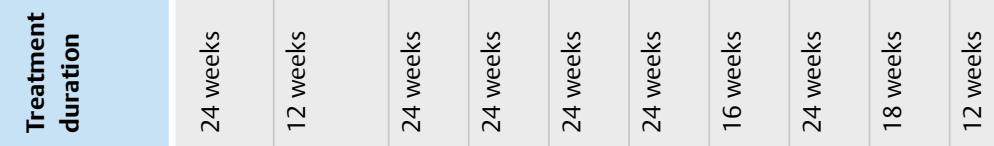

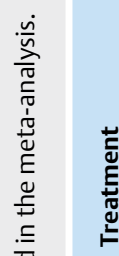

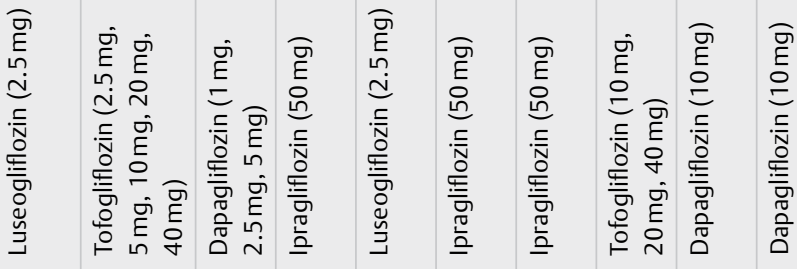

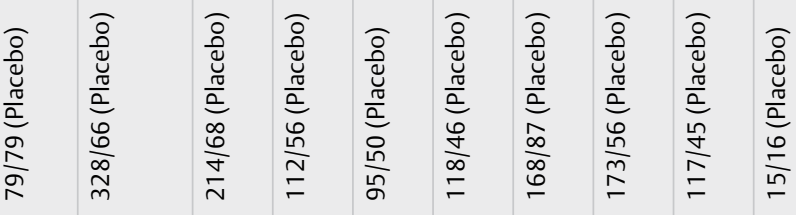

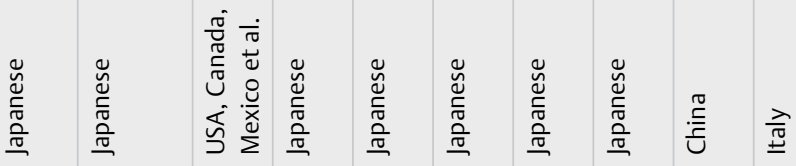

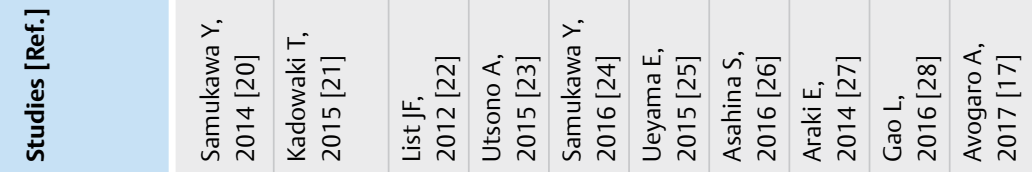




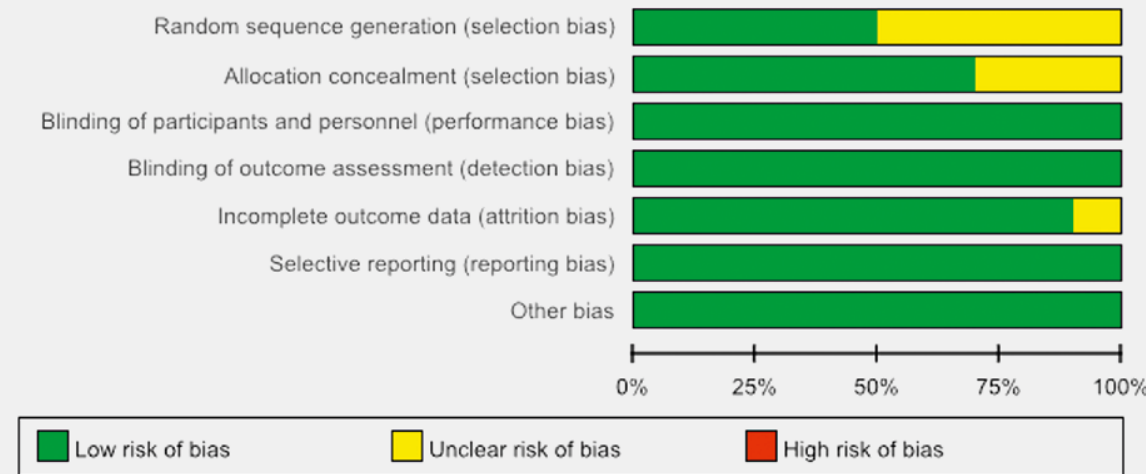

- Fig. 2 Study quality as estimated by Cochrane scores.

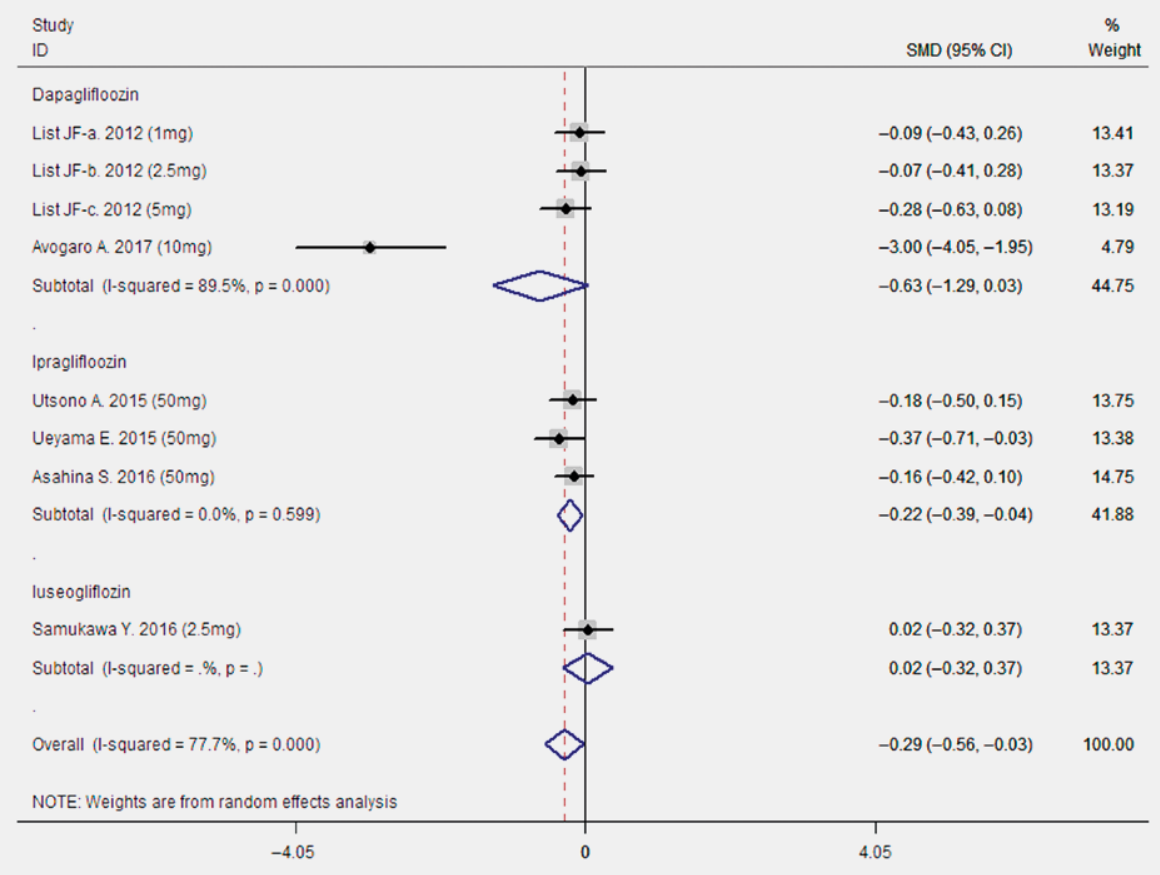

- Fig. 3 Meta-analysis of the effect of SGLT2i treatment versus placebo on serum leptin levels.

excretion and weight loss. Additionally, insulin resistance is related to increased circulating leptin and decreased serum adiponectin concentrations [31]. Our study indicated that use of SGLT2 inhibitor decreased circulating leptin while elevated circulating adiponectin, suggesting that SGLT2 inhibitor may improve insulin resistance through regulation of the circulating adipokines. It is worth noting that whether SGLT2 mediating concentrations of adipokines plays the primary role in improving systemic insulin sensitivity. Friedman et al. have reported that congenital leptin deficiency is related to hyperphagia, hyperlipidemia as well as insulin resistance [3]. Also, leptin replacement therapy can reverse liver and muscle insulin resistance in patients with severe lipodystrophy [8]. Regarding adiponectin, it improves insulin sensitivity by binding to Adi-
poR1 and AdipoR2 resulting in AMPK pathway activation [32]. Then AMPK accelerates cellular metabolism and stimulates glucose uptake and fatty acid oxidation, thereby bringing about an improvement of insulin sensitivity [33]. The study of Lodish et al. showed that treatment of adiponectin improved muscle insulin sensitivity in mice, consistent with decreased body weight [34]. SGLT2 inhibitor is likely to be involved in regulation of other types of adipokines. Whether the pooled effect of SGLT2 inhibitor on adipokines contributing to improved insulin resistance, or overall metabolic homeostasis, remains to be explored.

T2DM is positively associated with the incidence and mortality of cardiovascular disease, which is the leading cause of death in T2DM patients [35]. Some studies have shown that single and in- 


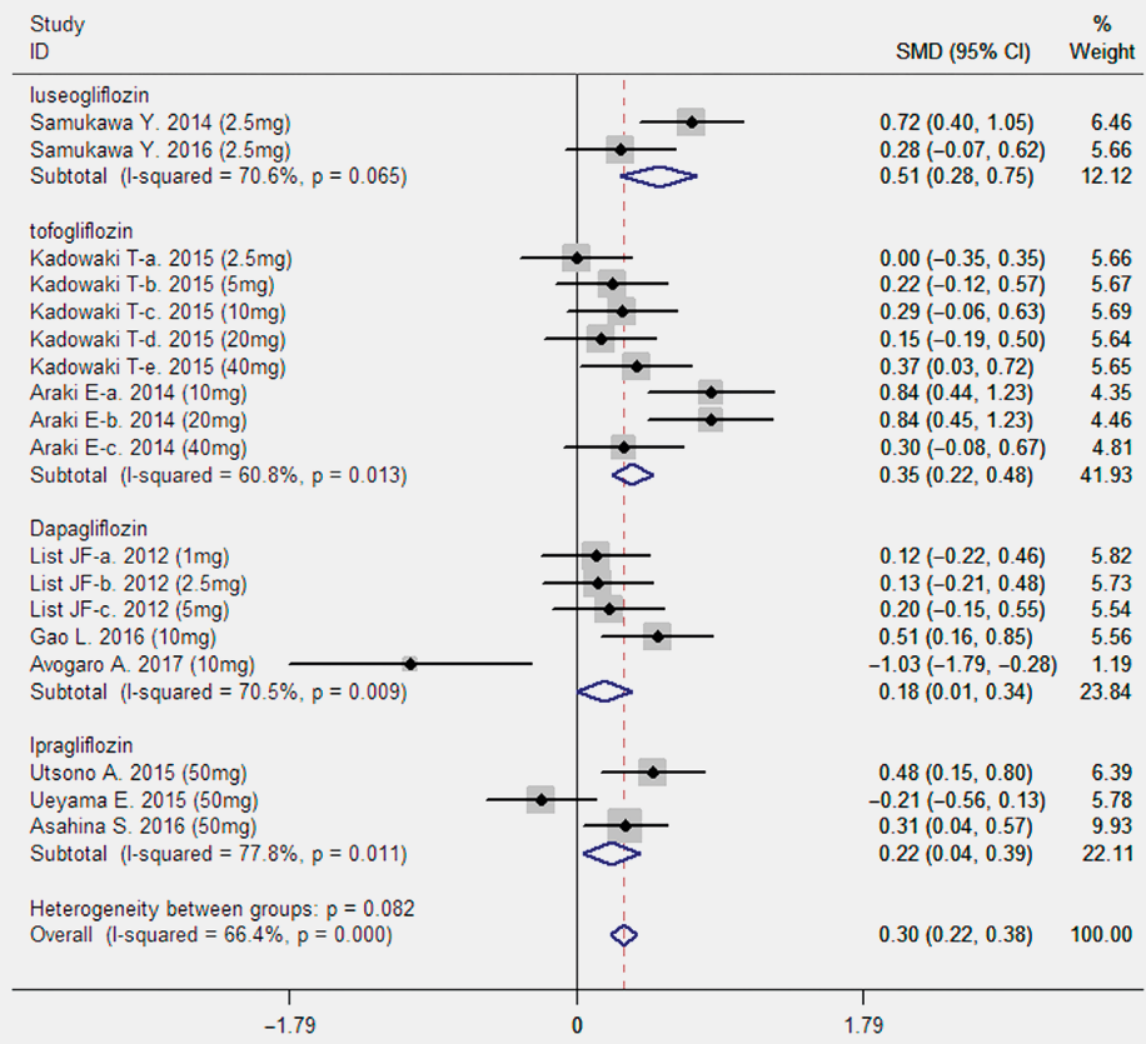

- Fig. 4 Meta-analysis of the effect of SGLT2i treatment versus placebo on serum adiponectin levels.

- Table 2 Subgroup analysis of included studies.

\begin{tabular}{|c|c|c|c|c|c|c|c|c|c|c|}
\hline \multirow[t]{2}{*}{ Groups } & \multirow[b]{2}{*}{$\mathbf{n}$} & \multicolumn{4}{|l|}{ Leptin } & \multicolumn{5}{|c|}{ Adiponectin } \\
\hline & & SMD (95\% Cl) & $\mathbf{p}$ & $\left.\right|^{2}(\%)$ & $\begin{array}{l}\text { p (hetero- } \\
\text { geneity) }\end{array}$ & $\mathbf{n}$ & SMD (95\% CI) & $\mathbf{p}$ & $\left.\right|^{2}(\%)$ & $\begin{array}{l}\text { p (hetero- } \\
\text { geneity) }\end{array}$ \\
\hline \multicolumn{11}{|l|}{ Age } \\
\hline$\geq 60$ years & 3 & $-0.94(-1.97,0.08)$ & 0.072 & 93.10 & 0 & 3 & $-0.24(-0.83,0.35)$ & 0.43 & 81.60 & 0.004 \\
\hline$<60$ years & 5 & $-0.15(-0.29,-0.01)$ & 0.035 & 0 & 0.926 & 15 & $0.36(0.23,0.48)$ & 0 & 50.70 & 0.013 \\
\hline \multicolumn{11}{|l|}{ BMI } \\
\hline$\geq 30$ & 4 & $-0.63(-1.29,0.03)$ & 0.060 & 89.50 & 0 & 9 & $0.14(-0.01,0.29)$ & 0.072 & 37.00 & 0.122 \\
\hline$<30$ & 4 & $-0.17(-0.32,-0.01)$ & 0.033 & 0 & 0.471 & 9 & $0.44(0.23,0.65)$ & 0 & 70.90 & 0.001 \\
\hline \multicolumn{11}{|l|}{ Duration } \\
\hline$\geq 24$ weeks & 6 & $-0.16(-0.30,-0.02)$ & 0.026 & 0 & 0.635 & 8 & $0.20(0.01,0.39)$ & 0.043 & 56.90 & 0.023 \\
\hline$<24$ weeks & 2 & $-1.53(-4.32,1.25)$ & 0.281 & 96.30 & 0 & 10 & $0.36(0.15,0.57)$ & 0.001 & 72.10 & 0 \\
\hline
\end{tabular}

tensive hypoglycemic therapy is not conducive to T2DM patients with cardiovascular risk [36, 37]. Therefore, cardiovascular benefits must be considered in the treatment of diabetes. Several studies have proven that SGLT2 therapy significantly reduced the risk of cardiovascular events $[15,16]$, but the underlying mechanisms are not yet clear. The effect of SGLT2 on adipokines may also be re- lated to the mechanism of cardioprotection. The peripheral effects of leptin include activation of inflammatory responses, oxidative stress, thrombosis and atherosclerosis, thereby resulting in endothelial dysfunction and atherosclerotic plaque [38, 39]. And leptin treatment was reported to attenuate atherosclerotic lesions in leptin-deficient low-density lipoprotein receptor mice [40]. Apart 
from leptin, adipokines including adiponectin, may be linked with the pathogenesis of cardiovascular disease. Low adiponectin levels are connected to increased risk of cardiovascular disease [41, 42]. Adiponectin protects against atherosclerosis through suppression of the expression of monocyte adhesion molecules and synthesis of inflammatory factors by inhibiting nuclear factor-kappa B $[11,43]$. Adiponectin also is known to target extracellular signal-regulated kinase and thus suppress proliferation of vascular smooth muscle cells [44]. Although it is unable to elucidate whether the changes of adipokines were directly regulated by SGLT2 inhibitors, these changes of leptin and adiponectin did have clear cardiovascular benefits. Based on above consideration, our study may partly explain the protective effect of SGLT2 on cardiovascular disease by regulation of circulating adipokines.

Several limitations of this study deserve some serious consideration. First, some confounding variables including age, BMI and control of glucose are difficult to avoid, which might affect the overall estimation. Second, some of the included studies have a small sample size, resulting in a high level of heterogeneity in circulating leptin and adiponectin concentration, which weaken the strength of the evidence of our study. Third, the number of studies regarding specific types of SGLT2 inhibitors is relatively small, and thereby have difficulty to explore the effect of each type of SGLT2 inhibitors on adipokines. Finally, it is uncertain whether the changes of adipokines are the direct effect of SGLT2 inhibitors, or whether they are secondary to the reduced body fat or the regulation of adipose tissue function. Therefore, more experiments should be carried out in the future to clarify the relationship between SGLT2 inhibitors use and changes of adipokines, and thereby further verify our findings.

\section{Conclusions}

SGLT2 inhibitor therapy was associated with a decreased circulating leptin levels and an increased circulating adiponectin levels, which might contribute to the beneficial effects of SGLT2 inhibitors on metabolic homeostasis, such as improved insulin resistance and reduced cardiovascular risk.

\section{Funding}

The work was supported by National Natural Science Foundation of China $(81570716,81670783,81770835)$ and Natural Science Foundation of Guangdong Province (2016A030313633, 2017A030313473).

\section{Conflict of Interest}

The authors declare that they have no conflict of interest.

\section{References}

[1] Ashcroft FM, Rorsman P. Diabetes mellitus and the beta cell: The last ten years. Cell 2012; 148: 1160-1171

[2] Waki H, Tontonoz P. Endocrine functions of adipose tissue. Annu Rev Pathol 2007; 2: 31-56
[3] Zhang Y, Proenca R, Maffei M et al. Positional cloning of the mouse obese gene and its human homologue. Nature 1994; 372: 425-432

[4] Farooqi IS, O'Rahilly S. 20 years of leptin: Human disorders of leptin action. J Endocrinol 2014; 223: T63-T70

[5] Ouchi N, Parker JL, Lugus J] et al. Adipokines in inflammation and metabolic disease. Nat Rev Immunol 2011; 11: 85-97

[6] Roszkowska-Gancarz M, Kurylowicz A, Polosak J et al. Functional polymorphisms of the leptin and leptin receptor genes are associated with longevity and with the risk of myocardial infarction and of type 2 diabetes mellitus. Endokrynol Pol 2014; 65: 11-16

[7] Romanowski M, Dziedziejko V, Maciejewska-Karlowska A et al. Adiponectin and leptin gene polymorphisms in patients with post-transplant diabetes mellitus. Pharmacogenomics 2015; 16 : 1243-1251

[8] Perry RJ, Petersen KF, Shulman GI. Pleotropic effects of leptin to reverse insulin resistance and diabetic ketoacidosis. Diabetologia 2016; 59: 933-937

[9] D'Souza AM, Neumann UH, Glavas MM et al. The glucoregulatory actions of leptin. Mol Metab 2017; 6: 1052-1065

[10] Yamauchi T, Kamon J, Ito $Y$ et al. Cloning of adiponectin receptors that mediate antidiabetic metabolic effects. Nature 2003; 423: 762-769

[11] Ouchi N, Kihara S, Arita Y et al. Adiponectin, an adipocyte-derived plasma protein, inhibits endothelial NF-kappaB signaling through a CAMP-dependent pathway. Circulation 2000; 102: 1296-1301

[12] Bouassida A, Chamari K, Zaouali M et al. Review on leptin and adiponectin responses and adaptations to acute and chronic exercise. $\mathrm{Br}$ J Sports Med 2010; 44: 620-630

[13] Katsiki N, Mikhailidis DP, Banach M. Leptin, cardiovascular diseases and type 2 diabetes mellitus. Acta Pharmacol Sin 2018; 39: 1176-1188

[14] Wilding J, Fernando K, Milne $\mathrm{N}$ et al. SGLT2 inhibitors in type 2 diabetes management: Key evidence and implications for clinical practice. Diabetes Ther 2018; 9: 1757-1773

[15] Jia X, Mehta PB, Ye Y et al. SGLT2 inhibitors and cardiovascular outcomes: Current perspectives and future potentials. Curr Diab Rep 2018; 18: 63

[16] Baptist G. The cardiovascular benefits associated with the use of sodium-glucose cotransporter 2 inhibitors - Real-World Data. Eur Endocrinol 2018; 14: 17-23

[17] Fadini GP, Bonora BM, Zatti G et al. Effects of the SGLT2 inhibitor dapagliflozin on HDL cholesterol, particle size, and cholesterol efflux capacity in patients with type 2 diabetes: A randomized placebo-controlled trial. Cardiovasc Diabetol 2017; 16: 42

[18] Kusunoki M, Natsume Y, Miyata T et al. Effects of Concomitant Administration of Sodium Glucose Co-transporter 2 Inhibitor with Insulin on Hemoglobin A1c, Body Mass Index and Serum Lipid Profile in Japanese Type 2 Diabetic Patients. Drug Res (Stuttg) 2018; 68: 669-672

[19] Ito D, Shimizu S, Inoue K et al. Comparison of ipragliflozin and pioglitazone effects on nonalcoholic fatty liver disease in patients with type 2 diabetes: A randomized, 24-week, open-label, active-controlled trial. Diabetes Care 2017; 40: 1364-1372

[20] Seino Y, Sasaki T, Fukatsu A et al. Efficacy and safety of luseogliflozin as monotherapy in Japanese patients with type 2 diabetes mellitus: $A$ randomized, double-blind, placebo-controlled, phase 3 study. Curr Med Res Opin 2014; 30: 1245-1255

[21] Ikeda S, Takano Y, Cynshi O et al. A novel and selective sodium-glucose cotransporter-2 inhibitor, tofogliflozin, improves glycaemic control and lowers body weight in patients with type 2 diabetes mellitus. Diabetes Obes Metab 2015; 17: 984-993

[22] Bailey C], Iqbal N, T'Joen C et al. Dapagliflozin monotherapy in drug-naive patients with diabetes: A randomized-controlled trial of low-dose range. Diabetes Obes Metab 2012; 14: 951-959 
[23] Kashiwagi A, Kazuta K, Goto K et al. Ipragliflozin in combination with metformin for the treatment of Japanese patients with type 2 diabetes: ILLUMINATE, a randomized, double-blind, placebo-controlled study. Diabetes Obes Metab 2015; 17: 304-308

[24] Haneda $M$, Seino $Y$, Inagaki $N$ et al. Influence of renal function on the 52-week efficacy and safety of the sodium glucose cotransporter 2 inhibitor luseogliflozin in Japanese Patients with type 2 diabetes mellitus. Clin Ther 2016; 38: 66-88.e20

[25] Kashiwagi A, Takahashi $\mathrm{H}$, Ishikawa $\mathrm{H}$ et al. A randomized, double-blind, placebo-controlled study on long-term efficacy and safety of ipragliflozin treatment in patients with type 2 diabetes mellitus and renal impairment: Results of the long-term ASP1941 safety evaluation in patients with type 2 diabetes with renal impairment (LANTERN) study. Diabetes Obes Metab 2015; 17: 152-160

[26] Ishihara H, Yamaguchi S, Nakao I et al. Efficacy and safety of ipragliflozin as add-on therapy to insulin in Japanese patients with type 2 diabetes mellitus (IOLITE): A multi-centre, randomized, placebo-controlled, double-blind study. Diabetes Obes Metab 2016; 18: 1207-1216

[27] Kaku K, Watada H, Iwamoto $Y$ et al. Efficacy and safety of monotherapy with the novel sodium/glucose cotransporter-2 inhibitor tofogliflozin in Japanese patients with type 2 diabetes mellitus: A combined Phase 2 and 3 randomized, placebo-controlled, double-blind, parallel-group comparative study. Cardiovasc Diabetol 2014; 13: 65

[28] Liao X, Wang X, Li H et al. Sodium-Glucose Cotransporter 2 (SGLT2) Inhibitor Increases Circulating Zinc-Alpha2-Glycoprotein Levels in Patients with Type 2 Diabetes. Sci Rep 2016; 6: 32887

[29] Ferrannini E, Muscelli E, Frascerra $S$ et al. Metabolic response to sodium-glucose cotransporter 2 inhibition in type 2 diabetic patients. J Clin Invest 2014; 124: 499-508

[30] Merovci A, Solis-Herrera C, Daniele G et al. Dapagliflozin improves muscle insulin sensitivity but enhances endogenous glucose production. J Clin Invest 2014; 124: 509-514

[31] Nicholson T, Church C, Baker DJ et al. The role of adipokines in skeletal muscle inflammation and insulin sensitivity. J Inflamm (Lond) 2018; 15: 9

[32] Tsuchida A, Yamauchi T, Ito Y et al. Insulin/Foxo1 pathway regulates expression levels of adiponectin receptors and adiponectin sensitivity. J Biol Chem 2004; 279: 30817-30822
[33] Hopkins TA, Ouchi N, Shibata R et al. Adiponectin actions in the cardiovascular system. Cardiovasc Res 2007; 74: 11-18

[34] Fruebis ], Tsao TS, Javorschi S et al. Proteolytic cleavage product of 30-kDa adipocyte complement-related protein increases fatty acid oxidation in muscle and causes weight loss in mice. Proc Natl Acad Sci USA 2001; 98: 2005-2010

[35] UK Prospective Diabetes Study (UKPDS) Group. Intensive blood-glucose control with sulphonylureas or insulin compared with conventional treatment and risk of complications in patients with type 2 diabetes (UKPDS 33). Lancet 1998; 352: 837-853

[36] Gerstein HC, Miller ME, Byington RP et al. Effects of intensive glucose lowering in type 2 diabetes. N Engl J Med 2008; 358: 2545-2559

[37] Duckworth W, Abraira C, Moritz T et al. Glucose control and vascular complications in veterans with type 2 diabetes. N Engl J Med 2009; 360: 129-139

[38] Perez-Perez A, Vilarino-Garcia T, Fernandez-Riejos P et al. Role of leptin as a link between metabolism and the immune system. Cytokine Growth Factor Rev 2017; 35: 71-84

[39] Liberale L, Bonaventura A, Vecchie A et al. The role of adipocytokines in coronary atherosclerosis. Curr Atheroscler Rep 2017; 19: 10

[40] Hoffmann A, Ebert T, Kloting $\mathrm{N}$ et al. Leptin dose-dependently decreases atherosclerosis by attenuation of hypercholesterolemia and induction of adiponectin. Biochim Biophys Acta 2016; 1862: 113-120

[41] Kishida K, Funahashi T, Shimomura I. Molecular mechanisms of diabetes and atherosclerosis: Role of adiponectin. Endocr Metab Immune Disord Drug Targets 2012; 12: 118-131

[42] Zhang H, Mo X, Hao Y et al. Adiponectin levels and risk of coronary heart disease: A meta-analysis of prospective studies. Am J Med Sci 2013; 345: 455-461

[43] Kobashi C, Urakaze M, Kishida M et al. Adiponectin inhibits endothelial synthesis of interleukin-8. Circ Res 2005; 97: 1245-1252

[44] Arita Y, Kihara S, Ouchi N et al. Adipocyte-derived plasma protein adiponectin acts as a platelet-derived growth factor-BB-binding protein and regulates growth factor-induced common postreceptor signal in vascular smooth muscle cell. Circulation 2002; 105: 2893-2898 